\title{
SINGULARITIES ON THE BASE OF A FANO TYPE FIBRATION
}

\author{
CAUCHER BIRKAR
}

\begin{abstract}
Let $f: X \rightarrow Z$ be a Mori fibre space. M ${ }^{c}$ Kernan conjectured that the singularities of $Z$ are bounded in terms of the singularities of $X$. Shokurov generalised this to pairs: let $(X, B)$ be a klt pair and $f: X \rightarrow Z$ a contraction such that $K_{X}+B \sim_{\mathbb{R}} 0 / Z$ and that the general fibres of $f$ are Fano type varieties; adjunction for fibre spaces produces a discriminant divisor $B_{Z}$ and a moduli divisor $M_{Z}$ on $Z$. it is then conjectured that the singularities of $\left(Z, B_{Z}+M_{Z}\right)$ are bounded in terms of the singularities of $(X, B)$. We prove Shokurov conjecture when $\left(F, \operatorname{Supp} B_{F}\right)$ belongs to a bounded family where $F$ is a general fibre of $f$ and $K_{F}+B_{F}=\left.\left(K_{X}+B\right)\right|_{F}$.
\end{abstract}

\section{INTRODUCTION}

We work over an algebraically closed field $k$ of characteristic zero. Let $X$ be a variety with klt singularities and $f: X \rightarrow Z$ a $K_{X}$-negative extremal contraction. When $f$ is a divisorial contraction, we can check that the singularities of $Z$ are as good as the singularities of $X$. More precisely, in terms of log discrepancies we have

$$
a(E, X, 0) \leq a(E, Z, 0)
$$

for every prime divisor $E$ on birational models of $X, Z$. If $f$ is a flipping contraction, and if $f^{+}: X^{+} \rightarrow Z$ is the positive side of the flip, then singularities of $X^{+}$are as good as the singularities of $X$. This is important since if we want to prove a statement about $X$ we can often translate it into a similar statement about $Z$ or $X^{+}$(eg, finite generation).

If $f$ is not birational, one would still like to understand the singularities on $Z$ although this is much more complicated. Beside being an interesting problem on its own, it is also important for inductive arguments. $M^{c}$ Kernan conjectured that the singularities of $Z$ are bounded in terms of the singularities of $X$, that is:

Conjecture $1.1\left(M_{d, \epsilon}\right)$. Let $d$ be a natural number and $\epsilon>0$ a real number. Then, there is a real number $\delta>0$ depending on $d, \epsilon$ satisfying the following: let $f: X \rightarrow Z$ be a $K_{X}$-negative extremal contraction such that

- $X$ is $\epsilon$-lc of dimension d and $\mathbb{Q}$-factorial, and

- $\operatorname{dim} X>\operatorname{dim} Z$.

Then $Z$ is $\delta$-lc.

Date: October 29, 2018.

2010 MSC: 14E30, 14J17. 
See 2.1 for the definition of $\epsilon$-lc singularities. When $d=1$ or $d=2$, the conjecture is trivial since $Z$ would be a smooth curve or just a point. Mori and Prokhorov [16, Theorem 1.2.7] proved the conjecture for $d=3$ and $\epsilon=1$ but with $X$ having terminal singularities: in this case one can take $\delta=1$. Much more recently, Alexeev and Borisov [2] proved the conjecture for toric morphisms of toric varieties.

Shokurov generalised the conjecture to the setting of pairs. Let $f: X \rightarrow Z$ be a contraction of normal varieties, and $(X, B)$ klt such that $K_{X}+B \sim_{\mathbb{R}} 0 / Z$. By a construction of Kawamata [12][13] we may write

$$
K_{X}+B \sim_{\mathbb{R}} f^{*}\left(K_{Z}+B_{Z}+M_{Z}\right)
$$

where $B_{Z}$ is called the discriminant part and $M_{Z}$ is called the moduli part. The discriminant part is canonically determined as a Weil $\mathbb{R}$-divisor by the singularities of $(X, B)$ and the fibres over codimension one points of $Z$; the moduli part is then automatically determined as an $\mathbb{R}$-linear equivalence class, in particular, $M_{Z}$ may be represented by many different Weil $\mathbb{R}$-divisors. See 2.6 for more details.

We are ready to state a refined version of Shokurov's conjecture.

Conjecture $1.2\left(S_{d, \epsilon, \mathcal{P}}\right)$. Let $d$ be a natural number, $\epsilon>0$ a real number, and $\mathcal{P}$ a set of couples. Then, there is a real number $\delta>0$ depending on $d, \epsilon, \mathcal{P}$ satisfying the following: let $(X, B)$ be a pair and $f: X \rightarrow Z$ a contraction such that

- $(X, B)$ is $\epsilon$-lc of dimension d,

- $K_{X}+B \sim_{\mathbb{R}} 0 / Z$,

- the general fibres $F$ of $f$ are of Fano type,

- $\left(F, \operatorname{Supp} B_{F}\right)$ is isomorphic in codimension one with some $\left(F^{\prime}, D_{F^{\prime}}\right) \in \mathcal{P}$ where $K_{F}+B_{F}=\left.\left(K_{X}+B\right)\right|_{F}$.

Then, we can choose an $\mathbb{R}$-divisor $M_{Z} \geq 0$ representing the moduli part so that $\left(Z, B_{Z}+M_{Z}\right)$ is $\delta$-lc.

See 2.1, 2.3, and 2.8 for the definition of general fibres, Fano type varieties, couples and their boundedness. A couple is essentially a pair but with no condition on singularities except normality. Note that unlike in Conjecture 1.1, $f$ is allowed to be a divisorial contraction, a flipping contraction, or a fibre type contraction. Also note that we are not assuming $X, Z$ to be projective although $f$ is projective. Mori and Prokhorov [17, Theorem 1.1] prove a result on weak del Pezzo fibrations in dimension 3 which is closely related to the conjecture when $d=3, \epsilon=1, \operatorname{dim} Z=1$, and $-K_{F}$ is nef and big.

Now we come to the main theorem of this paper.

Theorem 1.3. Shokurov Conjecture $S_{d, \epsilon, \mathcal{P}}$ holds if $\mathcal{P}$ is a bounded family of couples.

To prove the theorem, we use a recent result of Hacon, $\mathrm{M}^{\mathrm{c}}$ Kernan, and $\mathrm{Xu}$ [10, Theorem 1.3] on volumes of big log divisors. In view of the theorem, it is natural to consider interesting cases of bounded $\mathcal{P}$ (Corollaries 1.4 and 1.5) and 
to try to reduce the conjecture to the theorem when $\mathcal{P}$ is not bounded (proof of Corollary 1.6 and Remark 4.3).

Let $d$ be a natural number and $\epsilon, \lambda>0$ be real numbers. Consider the pairs $\left(F, B_{F}\right)$ satisfying:

- $\left(F, B_{F}\right)$ is $\epsilon$-lc and of dimension $\leq d-1$,

- $K_{F}+B_{F} \sim_{\mathbb{R}} 0$,

- $F$ is of Fano-type,

- each non-zero coefficient of $B_{F}$ is $\geq \lambda$.

Let $\mathcal{R}$ be the set of the couples $\left(F, \operatorname{Supp} B_{F}\right)$. It is expected that $\mathcal{R}$ is a bounded family. This boundedness is known when $d \leq 3$ (see Theorem 4.1). Actually, if one tries to prove the boundedness in any dimension, then Theorem 1.3 appears naturally (see Remark 4.2 for a discussion on this).

Corollary 1.4. Conjecture $S_{d, \epsilon, \mathcal{R}}$ holds for the above data $d, \epsilon, \mathcal{R}$ when $d \leq 3$.

Under some extra assumptions, the boundedness of $\mathcal{R}$ is known in any dimension. More precisely: let $d$ be a natural number, $\epsilon>0$ a real number, and $\Lambda \subset[0,1]$ a finite set of real numbers. Consider the pairs $\left(F, B_{F}\right)$ satisfying:

- $\left(F, B_{F}\right)$ is $\epsilon$-lc and of dimension $\leq d-1$,

- $K_{F}+B_{F} \sim_{\mathbb{R}} 0$,

- $F$ is projective and $-K_{F}$ is ample, i.e. $F$ is a Fano variety,

- the coefficients of $B_{F}$ belong to $\Lambda$.

Let $\mathcal{Q}$ be the set of the couples $\left(F, \operatorname{Supp} B_{F}\right)$. By [10, Corollary 1.7], $\mathcal{Q}$ is a bounded family.

Corollary 1.5. Conjecture $S_{d, \epsilon, \mathcal{Q}}$ holds for the above data $d, \epsilon, \mathcal{Q}$.

For surfaces we can verify $S_{d, \epsilon, \mathcal{P}}$ without boundedness assumptions:

Corollary 1.6. Conjecture $S_{2, \epsilon, \mathcal{P}}$ holds. More generally: Conjecture $S_{d, \epsilon, \mathcal{P}}$ holds for those $(X, B)$ and $f: X \rightarrow Z$ with $d \leq \operatorname{dim} Z+1$.

We say a few words about the proof of Theorem 1.3. The difficult part of the theorem is to deal with the discriminant part $B_{Z}$ since by applying a result of Ambro [3] we can control the moduli part $M_{Z}$ (actually we have to understand the discriminant b-divisor $\mathcal{B}_{Z}$ rather than just $B_{Z}$ ). By taking hyperplane sections of $Z$ one can reduce the problem to the case $\operatorname{dim} Z=1$. Here one is mainly concerned about bounding the multiplicities of each fibre of some fibration birational to $f$. Mori and Prokhorov [17, Theorem 1.1] do this by using the orbifold Riemann-Roch theorem for varieties of dimension 3 with terminal singularities. Unfortunately, this approach does not work in higher dimensions. Instead of Riemann-Roch, we use the boundedness of volumes of big log divisors [10, Theorem 1.3].

Shokurov has another approach to the theorem: as far as I understand he is trying to construct a compactified coarse moduli space for the log general fibres and then recover information about $f$ from the moduli space. Our approach is more direct and it does not rely on the existence of such a moduli space. However, the techniques developed in this paper might in fact be useful to construct 
such moduli spaces.

Acknowledgements: This work was supported by a grant from Leverhulme. Thanks to Yuri Prokhorov for useful discussions on his results with Mori.

\section{Preliminaries}

2.1. Pairs. We work over an algebraically closed field $k$ of characteristic zero: all the varieties and schemes are over $k$ unless stated otherwise. A sub-pair $(X, B)$ consists of a normal quasi-projective variety $X$ and a sub-boundary $B$, that is, an $\mathbb{R}$-divisor on $X$ with coefficients in $(-\infty, 1]$ such that $K_{X}+B$ is $\mathbb{R}$ Cartier. For a prime divisor $D$ on some birational model of $X$ with a nonempty centre on $X, a(D, X, B)$ denotes the $\log$ discrepancy. We say that $(X, B)$ is $\epsilon$-lc if $a(D, X, B) \geq \epsilon$ for every prime divisor $D$ on birational models of $X$ (if $B=0$, we also say that $X$ is $\epsilon$-lc). This is equivalent to the following: let $g: Y \rightarrow X$ be any projective birational morphism from a normal variety $Y$ and write $K_{Y}+B_{Y}:=g^{*}\left(K_{X}+B\right)$; then every coefficient of $B_{Y}$ is $\leq 1-\epsilon$.

We call a sub-pair $(X, B) l c$ if it is 0-lc. We call it $k l t$ if it is $\epsilon$-lc for some $\epsilon>0$. A sub-pair $(X, B)$ is called a pair if the coefficients of $B$ are non-negative: in this case we call $B$ a boundary. We refer to [14] for standard definitions and results on singularities of pairs and the log minimal model program.

Let $(X, B)$ be a lc sub-pair and $M \geq 0$ an $\mathbb{R}$-Cartier divisor. The lc threshold of $M$ with respect to $(X, B)$ is the largest real number $t$ so that $(X, B+t M)$ is lc.

A contraction $f: X \rightarrow Z$ is a projective morphism of quasi-projective varieties with $f_{*} \mathscr{O}_{X}=\mathscr{O}_{Z}$. A general fibre of $f$ is a fibre over a closed point belonging to some fixed open set $U \subset Z$. In practice, $U$ is understood from the context and we might shrink it without mention. If $(X, B)$ is a pair, a $\log$ general fibre of $(X, B)$ and $f$ is as $\left(F, B_{F}\right)$ where $F$ is a general fibre of $f$ and $K_{F}+B_{F}=\left.\left(K_{X}+B\right)\right|_{F}$.

2.2. Minimal models and Mori fibre spaces. Let $(X, B)$ be a lc pair, $\left(Y, B_{Y}\right)$ a $\mathbb{Q}$-factorial dlt pair, $X \rightarrow Z$ and $Y \rightarrow Z$ contractions, $\phi: X \rightarrow Y / Z$ a birational map such that $\phi^{-1}$ does not contract divisors, and $B_{Y}=\phi_{*} B$. Moreover, assume that

$$
a(D, X, B) \leq a\left(D, Y, B_{Y}\right)
$$

for any prime divisor $D$ on birational models of $X$ and assume that the strict inequality holds for any prime divisor $D$ on $X$ which is exceptional $/ Y$. We say that $\left(Y, B_{Y}\right)$ is a $\log$ minimal model of $(X, B)$ over $Z$ if $K_{Y}+B_{Y}$ is nef $/ Z$. On the other hand, we say that $\left(Y, B_{Y}\right)$ is a Mori fibre space of $(X, B)$ over $Z$ if there is a $K_{Y}+B_{Y}$-negative extremal contraction $Y \rightarrow Y^{\prime} / Z$ such that $\operatorname{dim} Y^{\prime}<\operatorname{dim} Y$. These definitions follow the traditional definitions of $\log$ minimal models and Mori fibre spaces. The corresponding definitions in [6] are more general but we do not need such generality in this paper. 
Assume that $(X, B)$ is a $\mathbb{Q}$-factorial klt pair and $f: X \rightarrow Z$ a contraction with $K_{X}+B$ or $B \mathrm{big} / Z$. Also assume that $C \geq 0,(X, B+C)$ is klt, and $K_{X}+B+C$ is nef $/ Z$. Then by [7], any LMMP $/ Z$ on $K_{X}+B$ with scaling of $C$ ends up with a log minimal model or a Mori fibre space over $Z$.

2.3. Fano type varieties. A projective variety $X$ is said to be of Fano type if there is a boundary $C$ such that $(X, C)$ is a klt pair and $-\left(K_{X}+C\right)$ is ample. Note that if there is another boundary $B$ with $K_{X}+B \sim_{\mathbb{R}} 0$, then $B$ is big because $-K_{X}$ is big.

Lemma 2.4. Assume that $\phi: X \rightarrow X^{\prime}$ is an isomorphism in codimension one between normal projective varieties. If $X$ is of Fano type, then $X^{\prime}$ is also of Fano type.

Proof. There is a boundary $C$ such that $(X, C)$ is a klt pair and $-\left(K_{X}+C\right)$ is ample. There is an $\mathbb{R}$-Cartier divisor $D$ such that $(X, C+D)$ is klt and $K_{X}+C+D \sim_{\mathbb{R}} 0$. Obviously, $D$ is ample. Now, $\left(X^{\prime}, C^{\prime}+D^{\prime}\right)$ is klt, $K_{X^{\prime}}+$ $C^{\prime}+D^{\prime} \sim_{\mathbb{R}} 0$, and $D^{\prime}$ is big where $C^{\prime}$ denotes the birational transform of $C$ (similar notation for the other divisors). We can write $D^{\prime} \sim_{\mathbb{R}} A^{\prime}+G^{\prime}$ where $A^{\prime}$ is ample and $G^{\prime} \geq 0$. Thus,

$$
\left(X^{\prime}, C^{\prime}+(1-t) D^{\prime}+t A^{\prime}+t G^{\prime}\right)
$$

is klt and

$$
K_{X^{\prime}}+C^{\prime}+(1-t) D^{\prime}+t A^{\prime}+t G^{\prime} \sim_{\mathbb{R}} 0
$$

if $t>0$ is sufficiently small. Put $\Delta^{\prime}=C^{\prime}+(1-t) D^{\prime}+t G^{\prime}$. Then, $\left(X^{\prime}, \Delta^{\prime}\right)$ is klt and $-\left(K_{X^{\prime}}+\Delta^{\prime}\right)$ is ample hence $X^{\prime}$ is also of Fano type.

2.5. B-divisors. Let $X$ be a normal variety. An $\mathbb{R}$-b-divisor $\mathcal{D}$ on $X$ is a collection of $\mathbb{R}$-divisors $D_{Y}$ for each birational contraction $Y \rightarrow X$ so that if we have a birational contraction $\pi: Y^{\prime} \rightarrow Y / X$, then $\pi_{*} D_{Y^{\prime}}=D_{Y}$. In birational geometry, one often has to consider all the resolutions of a variety $X$ in order to understand properties of a divisor on $X$. This naturally leads to b-divisors which were defined by Shokurov [19][21].

2.6. Adjunction for fibre spaces. Let $f: X \rightarrow Z$ be a contraction of normal varieties, and $(X, B)$ a klt sub-pair such that $K_{X}+B \sim_{\mathbb{R}} 0 / Z$, that is, we have an equivalence $K_{X}+B \sim_{\mathbb{R}} f^{*} N$ for some $\mathbb{R}$-Cartier $\mathbb{R}$-divisor $N$. By a construction of Kawamata [12][13] we have a decomposition

$$
N \sim_{\mathbb{R}} K_{Z}+B_{Z}+M_{Z}
$$

where $B_{Z}$ is defined using the singularities of $(X, B)$ and of the fibres of $f$. The part $B_{Z}$ is called the discriminant part and the part $M_{Z}$ is called the moduli part. More precisely, $B_{Z}$ is defined as follows: for each prime divisor $D$ on $Z$, let $t$ be the lc threshold of $f^{*} D$ over the generic point of $D$, with respect to the pair $(X, B)$; then let $(1-t)$ be the coefficient of $D$ in $B_{Z}$. The moduli part $M_{Z}$ is then determined as an $\mathbb{R}$-linear equivalence class. 
Consider a commutative diagram

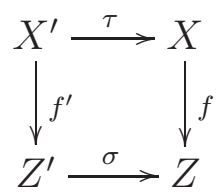

in which $X^{\prime}, Z^{\prime}$ are normal, $\sigma, \tau$ are birational contractions, and $f^{\prime}$ is a contraction. Let $K_{X^{\prime}}+B^{\prime}:=\tau^{*}\left(K_{X}+B\right)$. Using the relation $K_{X^{\prime}}+B^{\prime} \sim_{\mathbb{R}} 0 / Z^{\prime}$, we can similarly define a decomposition

$$
\sigma^{*} N \sim_{\mathbb{R}} K_{Z^{\prime}}+B_{Z^{\prime}}+M_{Z^{\prime}}
$$

which satisfies $B_{Z}=\sigma_{*} B_{Z^{\prime}}$ and $M_{Z} \sim_{\mathbb{R}} \sigma_{*} M_{Z^{\prime}}$. Putting all the $B_{Z^{\prime}}$ together for all the possible $Z^{\prime}$ determines an $\mathbb{R}$-b-divisor $\mathcal{B}_{Z}$. We could also choose the $M_{Z^{\prime}}$ consistently so that putting all the $M_{Z^{\prime}}$ together we get an $\mathbb{R}$-b-divisor $\mathcal{M}_{Z}$.

Now assume that $X, Z$ are projective and that $B$ is effective with rational coefficients. Kawamata [12][13] showed that if $Z^{\prime}$ is a sufficiently high resolution then $M_{Z^{\prime}}$ is a nef $\mathbb{Q}$-divisor. Following ideas of Kawamata [11], Ambro [3] proved that, perhaps after replacing $Z^{\prime}$ with a higher resolution, $M_{Z^{\prime}}$ satisfies a pullback property: for any other resolution $\pi: Z^{\prime \prime} \rightarrow Z^{\prime}$ we have $M_{Z^{\prime \prime}} \sim_{\mathbb{Q}}$ $\pi^{*} M_{Z^{\prime}}$. Moreover, he showed that $M_{Z^{\prime}}$ is the pullback of a nef and big divisor under some contraction $Z^{\prime} \rightarrow T$. We call such a $Z^{\prime}$ an Ambro model.

2.7. Volume of divisors. Let $X$ be a normal projective variety of dimension $d$ and $D$ an integral divisor on $X$. The volume of $D$ denoted by $\operatorname{vol}(D)$ is defined as

$$
\operatorname{vol}(D)=\limsup _{m \rightarrow+\infty} \frac{h^{0}\left(\mathscr{O}_{X}(m D)\right)}{m^{d} / d !}
$$

In some places we also use the notation $\operatorname{vol}\left(\mathscr{O}_{X}(D)\right)$ instead of $\operatorname{vol}(D)$.

If $D$ is a $\mathbb{Q}$-divisor, we can define $\operatorname{vol}(D)=\frac{1}{m^{d}} \operatorname{vol}(m D)$ for some $m>0$ with $m D$ being integral. If $D$ is not big, then it is obvious that $\operatorname{vol}(D)=0$. If $D$ is nef, then $\operatorname{vol}(D)=D^{d}$ which follows from the Riemann-Roch theorem. Ein-Lazarsfeld-Mustaţă-Nakamaye-Popa [15] treat this topic in detail.

Assume that $\Omega$ is a set of rational numbers satisfying the descending chain condition (DCC). Hacon-McKernan-Xu [10, Theorem 1.3] (see also [9]) proved that there is a number $\theta>0$ depending only on $d$ and $\Omega$ such that: if $(X, B)$ is a projective lc pair of dimension at most $d$, if the coefficients of $B$ belong to $\Omega$, and if $K_{X}+B$ is big, then $\operatorname{vol}\left(K_{X}+B\right) \geq \theta$. We will apply this in the proof of Proposition 3.1.

2.8. Couples and bounded families. A couple $\left(F, D_{F}\right)$ consists of a normal projective variety $F$ and a divisor $D_{F}$ on $F$ whose coefficients are all equal to 1, i.e. $D_{F}$ is a reduced divisor. The reason we call $\left(F, D_{F}\right)$ a couple rather than a pair is that we are concerned with $D_{F}$ rather than $K_{F}+D_{F}$ and we do not want to assume that $K_{F}+D_{F}$ is $\mathbb{Q}$-Cartier or that it has nice singularities.

Two couples $\left(F, D_{F}\right)$ and $\left(F^{\prime}, D_{F^{\prime}}\right)$ are isomorphic in codimension one if there is a birational isomorphism $\phi: F \rightarrow F^{\prime}$ which is an isomorphism in 
codimension one such that $\phi_{*} D_{F}=D_{F^{\prime}}$. The two couples are isomorphic if $\phi$ is an isomorphism.

We say that a set of couples $\mathcal{P}$ is a bounded family if there is a projective morphism $f: S \rightarrow T$ of Noetherian schemes over $k$ and a closed subscheme $D$ of $S$ such that for each $\left(F, D_{F}\right) \in \mathcal{P}$ there is a closed point $t \in T$ and an isomorphism between $\left(F, D_{F}\right)$ and $\left(S_{t}, \tilde{D}_{t}\right)$ where $S_{t}, D_{t}$ are the fibres over $t$ of the morphisms $S \rightarrow T$ and $D \rightarrow T$ respectively and $\tilde{D}_{t} \leq D_{t}$ : in particular, for such $t$ the scheme $S_{t}$ is a normal projective variety and the subscheme $D_{t}$ is a reduced divisor on $S_{t}$.

Lemma 2.9. Let $\mathcal{P}$ be a bounded family of couples. Then, there is a natural number $v$ depending only on $\mathcal{P}$ such that for any $\left(F, D_{F}\right) \in \mathcal{P}$ the volume $\operatorname{vol}\left(D_{F}\right) \leq v$.

Proof. As a general principle, the numerical invariants of a bounded family are bounded. We will give a detailed proof. Let $f: S \rightarrow T$ be as in the definition of bounded family of couples. Let $T^{\prime}$ be the reduced scheme associated to $T$ and let $S^{\prime}=S \times_{T} T^{\prime}$ and $D^{\prime}=D \times_{T} T^{\prime}$. Let $t$ be a closed point of $T$ and $S_{t}$ and $D_{t}$ the fibres over $t$ of the morphisms $S \rightarrow T$ and $D \rightarrow T$. These fibres are isomorphic to the corresponding fibres of the morphisms $S^{\prime} \rightarrow T^{\prime}$ and $D^{\prime} \rightarrow T^{\prime}$. Thus, we could assume from the beginning that $T$ is a reduced scheme. Moreover, we may assume that $T$ is affine by the Noetherian property.

Fix a closed embedding $S \rightarrow \mathbb{P}_{T}^{n} / T$ and let $\mathscr{O}_{S}(1)$ be the inverse image of $\mathscr{O}_{\mathbb{P}_{T}^{n}}(1)$. Since $S \rightarrow T$ is projective and $T$ affine, there is a coherent locally free sheaf $\mathscr{E}$ which is a direct sum of invertible sheaves of the form $\mathscr{O}_{S}(-m)$ with $m \gg 0$ admitting a surjective morphism $\mathscr{E} \rightarrow \mathscr{I}_{D}$ where $\mathscr{I}_{D}$ is the ideal sheaf of $D$ in $S$. This gives an exact sequence $\mathscr{E} \rightarrow \mathscr{O}_{S} \rightarrow \mathscr{O}_{D} \rightarrow 0$.

Fix a closed point $t \in T$ such that the fibre $S_{t}$ is a normal variety and that $D_{t}$ is a reduced divisor on $S_{t}$. Restricting the above exact sequence to $S_{t}$ we get an exact sequence $\mathscr{E}_{t} \rightarrow \mathscr{O}_{S_{t}} \rightarrow \mathscr{O}_{D_{t}} \rightarrow 0$. In particular, we have a surjection $\mathscr{E}_{t} \rightarrow \mathscr{I}_{D_{t}}$ where $\mathscr{I}_{D_{t}}$ is the ideal sheaf of $D_{t}$ in $S_{t}$. We then get an injection $\mathscr{I}_{D_{t}}^{\vee} \rightarrow \mathscr{E}_{t}^{\vee \vee}$ where for a coherent sheaf $\mathscr{N}$ on $S_{t}$ we define $\mathscr{N}^{\vee}:=$ $\mathscr{H}_{o m_{S_{t}}}\left(\mathscr{N}, \mathscr{O}_{S_{t}}\right)$. Let $U$ be the smooth locus of $S_{t}$. Then, $\left.\mathscr{I}_{D_{t}}\right|_{U}=\mathscr{O}_{U}\left(-D_{t}\right)$ hence $\left.\mathscr{I}_{D_{t}}^{\vee}\right|_{U}=\mathscr{O}_{U}\left(D_{t}\right)$. Since $\mathscr{E}_{t}^{\vee}$ is reflexive, the injection $\left.\left.\mathscr{I}_{D_{t}}^{\vee}\right|_{U} \rightarrow \mathscr{E}_{t} \vee\right|_{U}$ induces an injection $\mathscr{O}_{S_{t}}\left(D_{t}\right) \rightarrow \mathscr{E}_{t}$.

Let

$$
0 \rightarrow \mathscr{G}^{\prime} \rightarrow \mathscr{G} \rightarrow \mathscr{G}^{\prime \prime} \rightarrow 0
$$

be an exact sequence of coherent locally free sheaves on $S_{t}$ such that we are given an injection $\mathscr{O}_{S_{t}}\left(D_{t}\right) \rightarrow \mathscr{G}$. Then, by restricting to $U$ one can see that either the induced morphism $\mathscr{O}_{S_{t}}\left(D_{t}\right) \rightarrow \mathscr{G}^{\prime \prime}$ is injective or $\mathscr{O}_{S_{t}}\left(D_{t}\right)$ is mapped into the kernel of $\mathscr{G} \rightarrow \mathscr{G}^{\prime \prime}$ in which case we get an injection $\mathscr{O}_{S_{t}}\left(D_{t}\right) \rightarrow \mathscr{G}^{\prime}$.

By construction, $\mathscr{E}_{t} \vee$ is a coherent locally free sheaf which is a direct sum of sheaves of the form $\mathscr{O}_{S_{t}}(m)$ with $m \gg 0$. Applying the last paragraph we get an injection $\mathscr{O}_{S_{t}}\left(D_{t}\right) \rightarrow \mathscr{O}_{S_{t}}\left(m_{t}\right)$ for some $m_{t} \gg 0$ where there are only finitely many possibilities for $m_{t}$ for all $t$ as above. 
Now, since $T$ is reduced, by the generic flatness and stratification theorem, there are only finitely many possibilities for the Hilbert polynomial $\Phi_{t}$ of the fibre $S_{t}$. By definition, $\Phi_{t}(m)=\mathcal{X}\left(\mathscr{O}_{S_{t}}(m)\right)$. On the other hand, since $m_{t}$ is sufficiently large, $h^{i}\left(\mathscr{O}_{S_{t}}\left(m m_{t}\right)\right)=0$ for any $i, m>0$. Thus, $\Phi_{t}\left(m m_{t}\right)=$ $h^{0}\left(\mathscr{O}_{S_{t}}\left(m m_{t}\right)\right)$ for $m>0$ and this in turn implies that the volume

$$
\operatorname{vol}\left(\mathscr{O}_{S_{t}}\left(m_{t}\right)\right)=\limsup _{m \rightarrow+\infty} \frac{h^{0}\left(\mathscr{O}_{S_{t}}\left(m m_{t}\right)\right)}{m^{\operatorname{dim} S_{t}} / \operatorname{dim} S_{t} !}=\limsup _{m \rightarrow+\infty} \frac{\Phi_{t}\left(m m_{t}\right)}{m^{\operatorname{dim} S_{t}} / \operatorname{dim} S_{t} !}
$$

depends only on $\Phi_{t}$ and $m_{t}$. Since there are only finitely many possibilities for $\Phi_{t}$ and $m_{t}$, there is a natural number $v$ such that $\operatorname{vol}\left(\mathscr{O}_{S_{t}}\left(m_{t}\right)\right) \leq v$ for every $t$ as above. Thus, $\operatorname{vol}\left(D_{t}\right) \leq v$ because of the injection $\mathscr{O}_{S_{t}}\left(D_{t}\right) \rightarrow \mathscr{O}_{S_{t}}\left(m_{t}\right)$. By definition, each $\left(F, D_{F}\right) \in \mathcal{P}$ is isomorphic to some $\left(S_{t}, \tilde{D}_{t}\right)$ with $\tilde{D}_{t} \leq D_{t}$. Therefore,

$$
\operatorname{vol}\left(D_{F}\right)=\operatorname{vol}\left(\tilde{D}_{t}\right) \leq \operatorname{vol}\left(D_{t}\right) \leq v
$$

and we are done.

2.10. Intersection numbers. In certain cases we will apply the following lemma to compare intersection numbers on a variety which may not be proper.

Lemma 2.11. Let $h: Y \rightarrow Z$ be a contraction from a normal variety $Y$ of dimension d to a smooth curve $Z$. Assume that $L$ is a $\mathbb{Q}$-Cartier divisor on $Y$ which is nef/Z. Pick two distinct closed points $P, Q \in Z$ and write $h^{*} P=$ $\sum m_{i} T_{i}$ and $h^{*} Q=\sum n_{j} S_{j}$ where $T_{i}, S_{j}$ are the irreducible components. Then,

$$
\sum m_{i}\left(\left.L\right|_{T_{i}}\right)^{d-1}=\sum n_{j}\left(\left.L\right|_{S_{j}}\right)^{d-1}
$$

Proof. Take a resolution $\phi: W \rightarrow Y$. We can write $\phi^{*} h^{*} P=\sum m_{i} T_{i}^{\prime}+\sum l_{k} E_{k}$ where $E_{k}$ are prime exceptional divisors of $\phi$ and $T_{i}^{\prime}$ is the birational transform of $T_{i}$. Since $\left.\phi^{*} L\right|_{E_{k}}$ is not big, $\left(\left.\phi^{*} L\right|_{E_{k}}\right)^{d-1}=0$. On the other hand, since the induced morphism $T_{i}^{\prime} \rightarrow T_{i}$ is birational $\left(\left.\phi^{*} L\right|_{T_{i}^{\prime}}\right)^{d-1}=\left(\left.L\right|_{T_{i}}\right)^{d-1}$. Thus,

$$
\sum m_{i}\left(\left.L\right|_{T_{i}}\right)^{d-1}=\sum m_{i}\left(\left.\phi^{*} L\right|_{T_{i}^{\prime}}\right)^{d-1}+\sum l_{k}\left(\left.\phi^{*} L\right|_{E_{k}}\right)^{d-1}
$$

A similar equality holds for $\phi^{*} h^{*} Q$. Therefore, by replacing $X$ with $W$ and $L$ with $\phi^{*}$ we may assume that $X$ is already smooth.

By taking compactifications and then a resolution we can assume that there is a contraction $\bar{f}: \bar{Y} \rightarrow \bar{Z}$ of smooth projective varieties such that $Y \subset \bar{Y}$, $Z \subset \bar{Z}$, and that $\left.\bar{f}\right|_{Y}=f$. By replacing $\bar{f}$ with $f$ and $L$ with its closure in $\bar{Y}$, we may assume that $Y, Z$ are projective. Now, since $P-Q \equiv 0, h^{*} P \equiv h^{*} Q$ hence intersection with the 1-cycle $L^{d-1}$ would be the same which implies that

$$
\begin{gathered}
\sum m_{i}\left(\left.L\right|_{T_{i}}\right)^{d-1}=L^{d-1} \cdot\left(\sum m_{i} T_{i}\right)=L^{d-1} \cdot h^{*} P=L^{d-1} \cdot h^{*} Q \\
=L^{d-1} \cdot\left(\sum n_{j} S_{j}\right)=\sum n_{j}\left(\left.L\right|_{S_{j}}\right)^{d-1}
\end{gathered}
$$




\section{BOUNDING THE DISCRIMINANT B-DIVISOR}

In this section we will bound the coefficients of the discriminant b-divisor $\mathcal{B}_{Z}$ that is associated to the data in Theorem 1.3. First we deal with the discriminant divisor $B_{Z}$ and later we take care of the b-divisor.

Proposition 3.1. Assume that $d, \epsilon, \mathcal{P}$ are as in Conjecture $S_{d, \epsilon, \mathcal{P}}$ and that $\mathcal{P}$ is a bounded family of couples. Then, there is a real number $\delta>0$ depending only on $d, \epsilon, \mathcal{P}$ such that if $(X, B)$ and $f: X \rightarrow Z$ are as in the conjecture, then every coefficient of $B_{Z}$ is $\leq 1-\delta$.

By taking hyperplane sections on $Z$ we reduce the problem to the case $\operatorname{dim} Z=1$.

Lemma 3.2. Assume that Proposition 3.1 holds for the data $d-1, \epsilon, \mathcal{P}$. Then the proposition holds for the data $d, \epsilon, \mathcal{P}$ for those $(X, B)$ and $f: X \rightarrow Z$ with $\operatorname{dim} Z>1$.

Proof. Assume that $\delta>0$ is as in the proposition for the data $d-1, \epsilon, \mathcal{P}$. Let $(X, B)$ and $f: X \rightarrow Z$ be as in the proposition for the data $d, \epsilon, \mathcal{P}$, and assume that $\operatorname{dim} Z>1$. Let $D$ be a component of $B_{Z}$, and $t$ the lc threshold of $f^{*} D$ over the generic point of $D$ with respect to the pair $(X, B)$. We will show that $t \geq \delta$ which means that the coefficient of $D$ in $B_{Z}$ is at most $1-\delta$. By removing some codimension 2 closed subset of $Z$ we may assume that $Z$ is smooth. Moreover, we can assume that $\left(X, B+t f^{*} D\right)$ is lc whose lc centres all map onto $D$. By definition, $\left(X, B+t f^{*} D\right)$ has at least one lc centre.

Pick a general hyperplane section $H \subset Z$ (which would intersect $D$ ) and let $G=f^{*} H$. This ensures that $\left(X, B+G+t f^{*} D\right)$ is lc and that $(X, B+G)$ is $\epsilon$-lc in codimension $\geq 2$, that is, $a(P, X, B+G) \geq \epsilon$ for any prime divisor $P$ exceptional $/ X$. Letting

$$
K_{G}+B_{G}=\left.\left(K_{X}+B+G\right)\right|_{G}
$$

we get a klt pair $\left(G, B_{G}\right)$ and a contraction $g: G \rightarrow H$ which satisfy properties of the proposition for the data $d-1, \epsilon, \mathcal{P}$ : indeed, $\left(G, B_{G}\right)$ is $\epsilon$-lc of dimension $d-1, K_{G}+B_{G} \sim_{\mathbb{R}} 0 / H$, and the log general fibres of $g$ are among the log general fibres of $f$.

By further shrinking $Z$ around $D$ we can assume that $D_{H}:=D \cap H$ is irreducible. By construction, $\left(G, B_{G}+t g^{*} D_{H}\right)$ is lc. Moreover, if $V$ is a lc centre of $\left(X, B+t f^{*} D\right)$ then $V \cap G \neq \emptyset$ because $V$ is mapped onto $D$ and $G$ contains every fibre over points of $D_{H} \subset D$. Since $V$ and $G$ are both lc centres of $\left(X, B+G+t f^{*} D\right)$, by Ambro [4, Theorem 1.1] the intersection $V \cap G$ is a union of lc centres of $\left(X, B+G+t f^{*} D\right)$. Each lc centre of $\left(X, B+G+t f^{*} D\right)$ which sits inside $G$ is also a lc centre of $\left(G, B_{G}+t g^{*} D_{H}\right)$. Thus, $V \cap G$ is a union of lc centres of $\left(G, B_{G}+t g^{*} D_{H}\right)$. As $V \cap G$ maps onto $D_{H}$, there is a lc centre $W$ of $\left(G, B_{G}+t g^{*} D_{H}\right)$ which maps onto $D_{H}$. In particular, this means that $t$ is the lc threshold of $g^{*} D_{H}$ with respect to the pair $\left(G, B_{G}\right)$ over the generic point of $D_{H}$. By assumptions, $t \geq \delta$. Therefore, the coefficient of $D$ in $B_{Z}$ is at most $1-\delta$ and we are done. 
Proof. (of Proposition 3.1) Step 1. Let $d, \epsilon, \mathcal{P}$ be as in the proposition where $\mathcal{P}$ is a bounded family by assumption. We will apply induction so we can assume that the proposition holds for the data $d^{\prime}, \epsilon, \mathcal{P}$ if $d^{\prime}<d$. Let $(X, B)$ and $f: X \rightarrow Z$ be as in the proposition for the data $d, \epsilon, \mathcal{P}$. By Lemma 3.2, we may assume that $\operatorname{dim} Z=1$. By taking a $\mathbb{Q}$-factorialisation and applying Lemma 2.4 we may assume that $X$ is $\mathbb{Q}$-factorial.

Fix a closed point $D \in Z$. We will find a real number $\delta>0$ depending only on $d, \epsilon, \mathcal{P}$ such that the coefficient of $D$ in $B_{Z}$ is $\leq 1-\delta$. We can shrink $Z$ around $D$ if necessary. In particular, we can assume that each component of $B$ is either horizontal $/ Z$ or mapped to $D$.

Step 2. By decreasing $\epsilon$ if necessary we may assume that $\epsilon$ is rational. Put $b=1-\frac{\epsilon}{2}$. Since $(X, B)$ is $\epsilon$-lc, and since for any prime divisor $P$ on $X$ we have $\epsilon \leq a(P, X, B) \leq 1, b>0$. Let $\phi: W \rightarrow X$ be a log resolution of $\left(X, B+f^{*} D\right)$. Let $\left\{M_{i}\right\}$ be the set of components of $\phi^{*} f^{*} D$, let $\left\{M_{j}^{\prime}\right\}$ be the set of prime exceptional divisors of $\phi$ which do not belong to $\left\{M_{i}\right\}$, and let $\left\{M_{k}^{\prime \prime}\right\}$ be the set of components of the birational transform of $B$ which do not belong to $\left\{M_{i}\right\}$. Define

$$
\Delta_{W}=\sum M_{i}+\sum b M_{j}^{\prime}+\sum b_{k} M_{k}^{\prime \prime}
$$

where $b_{k}$ is the coefficient of $M_{k}^{\prime \prime}$ in the birational transform of $B$. Define

$$
\Gamma_{W}=\sum M_{i}+\sum b M_{j}^{\prime}+\sum b M_{k}^{\prime \prime}
$$

By construction, $\left(W, \Delta_{W}\right)$ and $\left(W, \Gamma_{W}\right)$ are both dlt, Supp $\Gamma_{W}=\operatorname{Supp} \Delta_{W}$, $\Gamma_{W}-\Delta_{W} \geq 0$,

$$
\left\lfloor\Gamma_{W}\right\rfloor=\left\lfloor\Delta_{W}\right\rfloor=\operatorname{Supp} \phi^{*} f^{*} D,
$$

each component of $\Gamma_{W}-\Delta_{W}$ is a component of the birational transform of $B$ and horizontal $/ Z$, and $\Gamma_{W}-\Delta_{W}$ and $\left\lfloor\Gamma_{W}\right\rfloor=\left\lfloor\Delta_{W}\right\rfloor$ have no common components. Also, note that the coefficients of $\Gamma_{W}$ belong to the set $\{b, 1\}$.

Write $K_{W}+B_{W}=\phi^{*}\left(K_{X}+B\right)$. Since $(X, B)$ is $\epsilon$-lc, each coefficient of $B_{W}$ is at most $1-\epsilon$. If $Q$ is a component of $B_{W}$ with positive coefficient, then $Q$ is exceptional/ $X$ or a component of the birational transform of $B$. In either case $Q$ is a component of $\Gamma_{W}$. So from $1-\epsilon<b$ we get $\Gamma_{W}-B_{W} \geq 0$ and that $\operatorname{Supp} \phi^{*} B \subseteq \operatorname{Supp}\left(\Gamma_{W}-B_{W}\right)$. Thus, $B_{W}+r \phi^{*} B \leq \Gamma_{W}$ if $r>0$ is sufficiently small. This implies that

$$
\phi^{*}\left(K_{X}+B+r B\right) \leq K_{W}+\Gamma_{W}
$$

hence $K_{W}+\Gamma_{W}$ is big/ $Z$ because $K_{X}+B+r B$ is big/ $Z$ which in turn follows from the assumptions that $K_{X}+B \sim_{\mathbb{R}} 0 / Z$ and that the general fibres of $f$ are of Fano type (see 2.3).

Step 3. Let $\Gamma=\phi_{*} \Gamma_{W}$. We can write

$$
K_{W}+\Gamma_{W}=\phi^{*}\left(K_{X}+\Gamma\right)+E_{W}
$$

where $E_{W}$ is exceptional/ $X$. Run the LMMP/X on $K_{W}+\Gamma_{W}$ with scaling of some ample divisor. By [6, Theorem 3.5], after finitely many steps we get a model $V$ on which $E_{V} \leq 0$. Let $g$ be the morphism $V \rightarrow Z$. Let $\left(G, \Gamma_{G}\right)$ be 
a log general fibre of $\left(V, \Gamma_{V}\right)$ and $g$, and let $\left(F, \Gamma_{F}\right)$ be the corresponding log general fibre of $(X, \Gamma)$ and $f$. Then,

$$
K_{G}+\Gamma_{G}=\psi^{*}\left(K_{F}+\Gamma_{F}\right)+E_{G}
$$

where $\psi$ is the morphism $G \rightarrow F$ and $E_{G}:=\left.E_{V}\right|_{G} \leq 0$.

By construction, $\operatorname{Supp} \Gamma=\operatorname{Supp} B$ over the generic point of $Z$ hence $\operatorname{Supp} \Gamma_{F}=$ $\operatorname{Supp} B_{F}$. By assumptions, the couple $\left(F, \operatorname{Supp} \Gamma_{F}\right)=\left(F, \operatorname{Supp} B_{F}\right)$ is isomorphic in codimension one with some couple $\left(F^{\prime}, D_{F^{\prime}}\right) \in \mathcal{P}$. By Lemma 2.9, the volume of $D_{F^{\prime}}$ is bounded by a number $v$ depending only on $\mathcal{P}$. Thus, the volume of $L_{F}:=\operatorname{Supp} \Gamma_{F}$ is also bounded by $v$ because $\operatorname{vol}\left(L_{F}\right)=\operatorname{vol}\left(D_{F^{\prime}}\right)$. On the other hand, since $K_{X}+B \sim_{\mathbb{R}} 0 / Z$, there is a rational boundary $B^{\prime}$ with the same support as $B$ such that $K_{X}+B^{\prime} \sim_{\mathbb{Q}} 0 / Z$ (see the approximation arguments in the proof of Theorem 1.3 below) from which we get

$$
K_{F}+\Gamma_{F} \leq K_{F}+L_{F}=K_{F}+B_{F}^{\prime}+L_{F}-B_{F}^{\prime} \sim_{\mathbb{Q}} L_{F}-B_{F}^{\prime} \leq L_{F}
$$

hence the volume of $K_{F}+\Gamma_{F}$ is bounded by $v$. Since $E_{G} \leq 0$, the volume of $K_{G}+\Gamma_{G}$ is also bounded by $v$.

Step 4. By construction, Supp $\left\lfloor\Gamma_{V}\right\rfloor=\operatorname{Supp} g^{*} D$. Since $g^{*} D \sim 0 / Z$, we can write

$$
K_{V}+\Gamma_{V} \sim_{\mathbb{Q}} K_{V}+C_{V} / Z
$$

where $C_{V} \leq \Gamma_{V},\left(V, C_{V}\right)$ is klt and $K_{V}+C_{V}$ is $\mathrm{big} / Z$. So, if we run the LMMP $/ Z$ on $K_{V}+\Gamma_{V}$ with scaling of some ample divisor, then it terminates with a model $Y$ on which $K_{Y}+\Gamma_{Y}$ is semi-ample/Z. Let $h$ denote the morphism $Y \rightarrow Z$.

Let $\left(H, \Gamma_{H}\right)$ be a log general fibre of $\left(Y, \Gamma_{Y}\right)$ and $h$, and let $\left(G, \Gamma_{G}\right)$ be the corresponding $\log$ general fibre of $\left(V, \Gamma_{V}\right)$ and $g$. By Step $3, \operatorname{vol}\left(K_{G}+\Gamma_{G}\right)$ is bounded. Since $\left(Y, \Gamma_{Y}\right)$ is a $\log$ minimal model of $\left(V, \Gamma_{V}\right)$ over $Z$,

$$
\operatorname{vol}\left(K_{H}+\Gamma_{H}\right)=\operatorname{vol}\left(K_{G}+\Gamma_{G}\right)
$$

which means that $\operatorname{vol}\left(K_{H}+\Gamma_{H}\right)$ is bounded by $v$ as well.

Step 5. Let $Y \rightarrow Y^{\prime}$ be the contraction $/ Z$ defined by $K_{Y}+\Gamma_{Y}$. Since $K_{Y}+\Gamma_{Y}$ is $\operatorname{big} / Z, Y \rightarrow Y^{\prime}$ is birational. Let $T$ be a component of $\operatorname{Supp}\left\lfloor\Gamma_{Y}\right\rfloor=\operatorname{Supp} h^{*} D$ that is not contracted over $Y^{\prime}$, that is,

$$
K_{T}+\Gamma_{T}:=\left.\left(K_{Y}+\Gamma_{Y}\right)\right|_{T}
$$

is big. Since the coefficients of $\Gamma_{Y}$ belong to the fixed finite set $\{b, 1\}$, the coefficients of $\Gamma_{T}$ belong to a DCC set $\Omega$ which only depend on $b$ and $d[18$, Corollary 3.10]. By [10, Theorem 1.3], there is a real number $\theta>0$ depending only on $d$ and $\Omega$ such that $\operatorname{vol}\left(K_{T}+\Gamma_{T}\right) \geq \theta$.

By Step 4, we can assume that $\operatorname{vol}\left(K_{H}+\Gamma_{H}\right) \leq v$ for every fibre $H$ of $h$ other than $h^{*} D$. Now, write $h^{*} D=\sum m_{i} T_{i}$ where $T_{i}$ are the irreducible components 
of $h^{*} D$. By Lemma 2.11, we get the following equalities of intersection numbers

$$
\begin{aligned}
\operatorname{vol}\left(K_{H}+\Gamma_{H}\right) & =\left(K_{H}+\Gamma_{H}\right)^{d-1} \\
& =\left(\left.\left(K_{Y}+\Gamma_{Y}\right)\right|_{H}\right)^{d-1} \\
& =\sum m_{i}\left(\left.\left(K_{Y}+\Gamma_{Y}\right)\right|_{T_{i}}\right)^{d-1} \\
& =\sum m_{i} \operatorname{vol}\left(K_{T_{i}}+\Gamma_{T_{i}}\right)
\end{aligned}
$$

Therefore if $K_{T_{i}}+\Gamma_{T_{i}}$ is big for some $i$, then

$$
\operatorname{vol}\left(K_{H}+\Gamma_{H}\right) \geq m_{i} \operatorname{vol}\left(K_{T_{i}}+\Gamma_{T_{i}}\right) \geq m_{i} \theta
$$

which implies that such $m_{i}$ are bounded by $\frac{v}{\theta}$. However, we do not get any bound for $m_{j}$ if $K_{T_{j}}+\Gamma_{T_{j}}$ is not big. We will try to get rid of such $T_{j}$.

Step 6. Run the LMMP $/ Y^{\prime}$ on $K_{Y}+\Delta_{Y}$ with scaling of $P_{Y}:=\Gamma_{Y}-\Delta_{Y}$. Note that $K_{Y}+\Gamma_{Y}$ is numerically trivial on each extremal ray contracted in the process since $K_{Y}+\Gamma_{Y} \equiv 0 / Y^{\prime}$. The LMMP terminates for reasons similar to Step 4. In some step of the LMMP, we arrive on a model on which the birational transform of $K_{Y}+\Gamma_{Y}-r P_{Y}$ is semi-ample/ $Y^{\prime}$ where $r>0$ is a small number. Replace $Y$ with that model. Since $K_{Y}+\Gamma_{Y}$ is semi-ample/Z, $K_{Y}+\Gamma_{Y}-r P_{Y}$ is also semi-ample $/ Z$ if we take $r$ to be sufficiently small.

Let $Y \rightarrow Y^{\prime \prime}$ be the contraction $/ Z$ defined by $K_{Y}+\Gamma_{Y}-r P_{Y}$. Since $r$ is sufficiently small, the map $Y^{\prime \prime} \rightarrow Y^{\prime}$ is actually a morphism. On the other hand, since, by Step 2, $P_{Y}$ and $\left\lfloor\Gamma_{Y}\right\rfloor$ have no common components, any component of $\left\lfloor\Gamma_{Y}\right\rfloor$ that is contracted over $Y^{\prime}$ is also contracted over $Y^{\prime \prime}$ : indeed if $T$ is a component of $\left\lfloor\Gamma_{Y}\right\rfloor$ that is contracted over $Y^{\prime}$, then $\left.\left(K_{Y}+\Gamma_{Y}\right)\right|_{T}$ is not big hence $\left.\left(K_{Y}+\Gamma_{Y}-r P_{Y}\right)\right|_{T}$ is also not big; so $T$ should be contracted over $Y^{\prime \prime}$ as well. Thus, by Step 5, the coefficient of any component of $h^{\prime \prime *} D$ is bounded by $\frac{v}{\theta}$ where $h^{\prime \prime}$ is the morphism $Y^{\prime \prime} \rightarrow Z$.

Step 7. Since $K_{Y^{\prime \prime}}+\Gamma_{Y^{\prime \prime}}$ and $K_{Y^{\prime \prime}}+\Gamma_{Y^{\prime \prime}}-r P_{Y^{\prime \prime}}$ are both $\mathbb{R}$-Cartier, $P_{Y^{\prime \prime}}$ is also $\mathbb{R}$-Cartier which in turn implies that $K_{Y^{\prime \prime}}+\Delta_{Y^{\prime \prime}}$ is $\mathbb{R}$-Cartier as well. In particular, $K_{Y^{\prime \prime}}+\Delta_{Y^{\prime \prime}}$ is lc. Let $\pi: N \rightarrow X$ and $\mu: N \rightarrow Y^{\prime \prime}$ be a common resolution. Since $(X, B)$ is $\epsilon$-lc, we can write

$$
K_{Y^{\prime \prime}}+B_{Y^{\prime \prime}}:=\mu_{*} \pi^{*}\left(K_{X}+B\right)
$$

where each coefficient of $B_{Y^{\prime \prime}}$ is $\leq 1-\epsilon$. On the other hand, if we write

$$
K_{W}+B_{W}=\phi^{*}\left(K_{X}+B\right)
$$

as in Step 2 then $B_{W}+\epsilon\left\lfloor\Delta_{W}\right\rfloor \leq \Delta_{W}$ which implies that $B_{Y^{\prime \prime}}+\epsilon\left\lfloor\Delta_{Y^{\prime \prime}}\right\rfloor \leq \Delta_{Y^{\prime \prime}}$ hence

$$
K_{Y^{\prime \prime}}+B_{Y^{\prime \prime}}+\epsilon\left\lfloor\Delta_{Y^{\prime \prime}}\right\rfloor \leq K_{Y^{\prime \prime}}+\Delta_{Y^{\prime \prime}}
$$

Moreover, $\left\lfloor\Delta_{Y^{\prime \prime}}\right\rfloor=\operatorname{Supp} h^{\prime \prime *} D$.

By Steps 5 and 6 , we can write $h^{\prime \prime *} D=\sum m_{i} T_{i}$ where the $m_{i}$ are all bounded by $\frac{v}{\theta}$. This implies that there is a real number $\delta>0$ depending only on $d, \epsilon, \mathcal{P}$ such that

$$
K_{Y^{\prime \prime}}+B_{Y^{\prime \prime}}+\delta h^{\prime \prime *} D \leq K_{Y^{\prime \prime}}+\Delta_{Y^{\prime \prime}}
$$


Therefore, $K_{Y^{\prime \prime}}+B_{Y^{\prime \prime}}+\delta h^{\prime \prime *} D$ is lc which in turn implies that $K_{X}+B+\delta f^{*} D$ is lc because

$$
\pi^{*}\left(K_{X}+B+\delta f^{*} D\right)=\mu^{*}\left(K_{Y^{\prime \prime}}+B_{Y^{\prime \prime}}+\delta h^{\prime \prime *} D\right)
$$

where we use the fact that $K_{X}+B \sim_{\mathbb{R}} 0 / Z$. Note however that $B_{Y^{\prime \prime}}+\delta h^{\prime \prime *} D$ is not necessarily effective but it is a sub-boundary. Finally, if $t$ is the lc threshold of $f^{*} D$ with respect to $(X, B)$, then we have $t \geq \delta$. In other words, the coefficient of $D$ in $B_{Z}$ is at most $1-\delta$ and this completes the proof of the proposition.

Next we bound the coefficients of the discriminant b-divisor $\mathcal{B}_{Z}$ (see 2.6 for definitions). But first we need a couple of lemmas.

Lemma 3.3. Assume that $\epsilon>\epsilon^{\prime}>0$. Let $(X, B)$ be a $\mathbb{Q}$-factorial $\epsilon$-lc pair and $f: X \rightarrow Z$ a contraction such that $K_{X}+B \sim_{\mathbb{R}} 0 / Z$ and $B$ is big/Z. Then, there are normal projective varieties $X^{\prime} \supseteq X$ and $Z^{\prime} \supseteq Z$ and a contraction $f^{\prime}: X^{\prime} \rightarrow Z^{\prime}$ such that

- $\left(X^{\prime}, B^{\prime}\right)$ is $\mathbb{Q}$-factorial $\epsilon^{\prime}$-lc and $K_{X^{\prime}}+B^{\prime} \sim_{\mathbb{R}} 0 / Z^{\prime}$,

- $\left.\left(K_{X^{\prime}}+B^{\prime}\right)\right|_{X}=K_{X}+B$, and $\left.f^{\prime}\right|_{X}=f$.

Proof. We can compactify the morphism $f$ to a morphism $\bar{f}: \bar{X} \rightarrow \bar{Z}$ where $\bar{X}$ and $\bar{Z}$ are normal projective varieties. Let $\phi: W \rightarrow \bar{X}$ be a $\log$ resolution and let $B_{W}=\bar{B}^{\sim}+\left(1-\epsilon^{\prime}\right) E$ where $E$ is the reduced exceptional divisor of $\phi, \bar{B}$ is the closure of $B$ in $\bar{X}$, and $\bar{B}^{\sim}$ is the birational transform of $\bar{B}$.

Run the LMMP $/ \bar{X}$ on $K_{W}+B_{W}$ with scaling of some ample divisor. We get a model $V$ on which $K_{V}+B_{V}$ is nef $/ \bar{X}$. Since $(X, B)$ is $\epsilon$-lc, over $X$, we can write $K_{W}+B_{W} \equiv G$ where $G$ is effective and exceptional. So, the LMMP contracts every component of $G$ over $X$ and since $X$ is $\mathbb{Q}$-factorial, $V \rightarrow \bar{X}$ is an isomorphism over $X$. Now, run the LMMP $/ \bar{Z}$ on $K_{V}+B_{V}$ with scaling of some ample divisor. Since $B_{V}$ is big $/ \bar{Z}$, the LMMP terminates with a model $X^{\prime}$ on which $K_{X^{\prime}}+B^{\prime}$ is semi-ample $/ \bar{Z}$.

Let $f^{\prime}: X^{\prime} \rightarrow Z^{\prime} \bar{Z}$ be the contraction defined by $K_{X^{\prime}}+B^{\prime}$. Since $V \rightarrow \bar{X}$ is an isomorphism over $X$ and $K_{X}+B \sim_{\mathbb{R}} 0 / Z$, the map $V \rightarrow X^{\prime}$ is an isomorphism over $Z$ hence the morphism $X^{\prime} \rightarrow Z^{\prime}$ coincides with $X \rightarrow Z$ over $Z$. By construction, $\left(X^{\prime}, B^{\prime}\right)$ and $f^{\prime}$ satisfy all the properties of the lemma.

Lemma 3.4. Let $(X, B)$ be a klt pair and $f: X \rightarrow Z$ a contraction such that $K_{X}+B \sim_{\mathbb{R}} 0 / Z$ and $B$ is big/Z. Assume that $D$ is a component of the discriminant b-divisor $\mathcal{B}_{Z}$ with positive coefficient. Then, there is an extremal contraction $Z^{\prime \prime} \rightarrow Z$ which extracts $D$.

Proof. By assumptions, there is a birational contraction $g: Z^{\prime} \rightarrow Z$ such that $D$ is a component of $B_{Z^{\prime}}$ with positive coefficient. By taking a $\mathbb{Q}$-factorialisation we may assume that $X$ is $\mathbb{Q}$-factorial. By Lemma 3.3, we may assume that $X, Z$ are projective. Assume that $B$ has rational coefficients. We will argue as in [3]. We may assume that $Z^{\prime}$ is an Ambro model and that $\left(Z^{\prime}, B_{Z^{\prime}}\right)$ is $\log$ smooth. In particular, $M_{Z^{\prime}}$ is the pullback of a nef and big divisor via some contraction $Z^{\prime} \rightarrow T$. Since $\left(Z^{\prime}, B_{Z^{\prime}}\right)$ is a klt sub-pair we can pick $M_{Z^{\prime}}$ so that 
$M_{Z^{\prime}} \geq 0$ and that $\left(Z^{\prime}, B_{Z^{\prime}}+M_{Z^{\prime}}\right)$ is again a klt sub-pair. Put $M_{Z}=g_{*} M_{Z^{\prime}}$. Then, $\left(Z, B_{Z}+M_{Z}\right)$ is a klt pair because

$$
K_{Z^{\prime}}+B_{Z^{\prime}}+M_{Z^{\prime}}=g^{*}\left(K_{Z}+B_{Z}+M_{Z}\right)
$$

Now, $a\left(D, Z, B_{Z}+M_{Z}\right)<1$ hence there is an extremal contraction $Z^{\prime \prime} \rightarrow Z$ which extracts $D$.

If $B$ is not a rational boundary, we can approximate $B$ with rational boundaries (cf. Fujino-Gongyo [8, Theorem 3.1]). More precisely, using Shokurov's polytopes [19], we can find a rational boundary $C$ sufficiently close to $B$ such that $(X, C)$ is klt, $K_{X}+C \sim_{\mathbb{R}} 0 / Z, C$ is big/Z, and the coefficient of $D$ in the discriminant b-divisor $\mathcal{C}_{Z}$ is positive. Now apply the arguments above for the case of rational boundaries.

Proposition 3.5. Assume that $d, \epsilon, \mathcal{P}$ are as in Conjecture $S_{d, \epsilon, \mathcal{P}}$ and that $\mathcal{P}$ is a bounded family of couples. Then, there is a real number $\delta>0$ depending only on $d, \epsilon, \mathcal{P}$ such that if $(X, B)$ and $f: X \rightarrow Z$ are as in the conjecture, then every coefficient of $\mathcal{B}_{Z}$ is $\leq 1-\delta$.

Proof. Pick $\epsilon^{\prime} \in(0, \epsilon)$ and let $\delta \in(0,1)$ be as in Proposition 3.1 for the data $d, \epsilon^{\prime}, \mathcal{P}$. Let $(X, B)$ and $f: X \rightarrow Z$ be as in Conjecture $S_{d, \epsilon, \mathcal{P}}$. By taking a $\mathbb{Q}$-factorialisation, we may assume that $X$ is $\mathbb{Q}$-factorial. This does not affect $\mathcal{B}_{Z}$. Assume that some component $E$ of $\mathcal{B}_{Z}$ has coefficient larger than $1-\delta$. We will derive a contradiction.

By our choice of $\delta$, every coefficient of $B_{Z}$ is $\leq 1-\delta$, so $E$ is exceptional $/ Z$. Since the general fibres of $f$ are of Fano type, $B$ is big/ $Z$. By Lemma 3.4, there is an extremal contraction $g: Z^{\prime \prime} \rightarrow Z$ such that $E$ is the only exceptional divisor of $g$. Now $B_{Z^{\prime \prime}} \geq 0$ and the coefficient of $E$ in $B_{Z^{\prime \prime}}$ is larger than $1-\delta$. To get a contradiction with Proposition 3.1 we need to construct a suitable fibration over $Z^{\prime \prime}$.

Let $\phi: W \rightarrow X$ be a $\log$ resolution so that the induced rational map $W \rightarrow Z^{\prime \prime}$ is a morphism. Let

$$
\Delta_{W}:=B^{\sim}+\left(1-\epsilon^{\prime}\right) G
$$

where $B^{\sim}$ is the birational transform of $B$ and $G$ is the reduced exceptional divisor of $\phi$. The pair $\left(W, \Delta_{W}\right)$ is $\epsilon^{\prime}$-lc and we can write

$$
K_{W}+\Delta_{W}=\phi^{*}\left(K_{X}+B\right)+C
$$

where $C \geq 0$ and $\operatorname{Supp} C=\operatorname{Supp} G$. In particular, if we run an LMMP on $K_{W}+\Delta_{W}$ over $X$ (or some open subset of $X$ ), then the LMMP terminates with $X$ (respectively with that open subset).

Let $T$ be the graph of the rational map $X \rightarrow Z^{\prime \prime}$, that is, $T$ is the closure in $X \times Z^{\prime \prime}$ of the graph of $X_{0} \rightarrow Z^{\prime \prime}$ where $X_{0} \subseteq X$ is the domain of $X \rightarrow Z^{\prime \prime}$. Since $W$ maps to both $X$ and $Z^{\prime \prime}$ we get an induced morphism $W \rightarrow T$. Let $U \subset Z$ be a non-empty open set over which $Z^{\prime \prime} \rightarrow Z$ is an isomorphism. Run an LMMP $/ T$ on $K_{W}+\Delta_{W}$ with scaling of some ample divisor. We end up with a model $Y$ on which $K_{Y}+\Delta_{Y}$ is nef/T. Since $f^{-1} U \subseteq X_{0}$, the morphism $T \rightarrow X$ is an isomorphism over $U$. So, the morphism $Y \rightarrow T$ is also an isomorphism over $U$ by the last paragraph. Since $K_{X}+B \sim_{\mathbb{R}} 0 / Z, K_{Y}+\Delta_{Y} \sim_{\mathbb{R}} 0$ over $U$. 
Now, run an LMMP $/ Z^{\prime \prime}$ on $K_{Y}+\Delta_{Y}$ with scaling of some ample divisor which ends up with a model $X^{\prime}$ on which $K_{X^{\prime}}+\Delta_{X^{\prime}}$ is semi-ample/ $Z^{\prime \prime}$ because $\Delta_{Y}$ is $\operatorname{big} / Z^{\prime \prime}$. Since $K_{Y}+\Delta_{Y}$ is nef over $U$, the LMMP does not modify $Y$ over $U$, that is, $Y \rightarrow X^{\prime}$ is an isomorphism over $U$.

Let $f^{\prime}: X^{\prime} \rightarrow Z^{\prime}$ be the contraction $/ Z^{\prime \prime}$ defined by $K_{X^{\prime}}+\Delta_{X^{\prime}}$. Then, the $\operatorname{map} Z^{\prime} \rightarrow Z^{\prime \prime}$ is birational. Moreover, $\left(X^{\prime}, \Delta_{X^{\prime}}\right)$ and $f^{\prime}: X^{\prime} \rightarrow Z^{\prime}$ satisfy the assumptions of Proposition 3.1 for the data $d, \epsilon^{\prime}, \mathcal{P}$, that is, $\left(X^{\prime}, \Delta_{X^{\prime}}\right)$ is $\epsilon^{\prime}$-lc of dimension $d, K_{X^{\prime}}+\Delta_{X^{\prime}} \sim_{\mathbb{R}} 0 / Z^{\prime}$, and the log general fibres of $\left(X^{\prime}, \Delta_{X^{\prime}}\right)$ and $f^{\prime}$ are the same as the log general fibres of $(X, B)$ and $f$.

Let $\Delta_{Z^{\prime}}$ be the discriminant on $Z^{\prime}$ associated to $K_{X^{\prime}}+\Delta_{X^{\prime}}$ and the fibration $f^{\prime}$. By Proposition 3.1, the coefficients of $\Delta_{Z^{\prime}}$ are at most $1-\delta$. On the other hand, let $B_{Z^{\prime}}$ be the discriminant on $Z^{\prime}$ associated to $K_{X}+B$ and the fibration $f$. It is enough to show that the coefficient of $E$ in $B_{Z^{\prime}}$ is not bigger than the coefficient of $E$ in $\Delta_{Z^{\prime}}$.

Let $\pi: V \rightarrow X$ and $\mu: V \rightarrow X^{\prime}$ be a common resolution and let

$$
M=K_{X^{\prime}}+\Delta_{X^{\prime}}-\mu_{*} \pi^{*}\left(K_{X}+B\right)
$$

As mentioned above we have

$$
K_{W}+\Delta_{W}-\phi^{*}\left(K_{X}+B\right)=C \geq 0
$$

Now $M$ is just the pushdown of $K_{W}+\Delta_{W}-\phi^{*}\left(K_{X}+B\right)$ via the rational map $W \rightarrow X^{\prime}$. Therefore, $M \geq 0$. From $K_{X}+B \sim_{\mathbb{R}} 0 / Z$ we get

$$
\pi^{*}\left(K_{X}+B\right)=\mu^{*} \mu_{*} \pi^{*}\left(K_{X}+B\right)
$$

and this combined with $M \geq 0$ results in

$$
\begin{gathered}
\mu^{*}\left(K_{X^{\prime}}+\Delta_{X^{\prime}}\right)-\pi^{*}\left(K_{X}+B\right) \\
=\mu^{*}\left(K_{X^{\prime}}+\Delta_{X^{\prime}}\right)-\mu^{*} \mu_{*} \pi^{*}\left(K_{X}+B\right)=\mu^{*} M \geq 0
\end{gathered}
$$

Since the coefficient of $E$ in $\Delta_{Z^{\prime}}$ is at most $1-\delta$, over the generic point of $E$ the log divisor $K_{X^{\prime}}+\Delta_{X^{\prime}}+\delta f^{\prime *} E$ is lc. This implies that

$$
\mu^{*}\left(K_{X^{\prime}}+\Delta_{X^{\prime}}+\delta f^{\prime *} E\right)=\mu^{*}\left(K_{X^{\prime}}+\Delta_{X^{\prime}}\right)+\mu^{*} \delta f^{\prime *} E
$$

is lc over the generic point of $E$ which in turn implies that

$$
\pi^{*}\left(K_{X}+B\right)+\delta \mu^{*} f^{*} E
$$

is also lc over the generic point of $E$. Therefore, the coefficient of $E$ in $B_{Z^{\prime}}$ is at most $1-\delta$. This gives a contradiction. Thus, every coefficient of $\mathcal{B}_{Z}$ is at most $1-\delta$.

\section{Proof of the MAIN ReSUlts}

Proof. (of Theorem 1.3) Let $(X, B)$ and $f: X \rightarrow Z$ be as in Conjecture $S_{d, \epsilon, \mathcal{P}}$. If $f$ is birational, then $B_{Z}=f_{*} B$ and if we take $M_{Z}=0$, then $K_{X}+B=f^{*}\left(K_{Z}+\right.$ $\left.B_{Z}\right)$ and $\left(Z, B_{Z}\right)$ is $\epsilon$-lc. So, $\delta=\epsilon$ works in this case. We can then assume that $f$ is not birational. By taking a $\mathbb{Q}$-factorialisation we may assume that $X$ is $\mathbb{Q}$-factorial. The log general fibres may change but only by an isomorphism in 
codimension one. Pick $\epsilon^{\prime} \in(0, \epsilon)$. By Lemma 3.3, we can replace $X, Z$ with projective varieties so that $(X, B)$ and $f: X \rightarrow Z$ satisfy the assumptions of Conjecture $S_{d, \epsilon^{\prime}, \mathcal{P}}$ (note that $\epsilon$ is replaced by $\epsilon^{\prime}$ ).

First assume that the coefficients of $B$ are rational numbers. Let $\delta^{\prime}>0$ be the number given by Proposition 3.5 for the data $d, \epsilon^{\prime}, \mathcal{P}$, and pick some $\delta \in\left(0, \delta^{\prime}\right)$. Let $g: Z^{\prime} \rightarrow Z$ be an Ambro model of $K_{X}+B$ and $f: X \rightarrow Z$ as defined in 2.6. Since $Z^{\prime}$ is an Ambro model, $M_{Z^{\prime}}$ is nef and good, that is, it is the pullback of a nef and big $\mathbb{Q}$-divisor $M_{T}$ via some contraction $\pi: Z^{\prime} \rightarrow T$. We can write $M_{T} \sim_{\mathbb{Q}} A_{T}+L_{T}$ where $A_{T}$ is an ample $\mathbb{Q}$-divisor and $L_{T} \geq 0$. Put $A_{Z^{\prime}}=\pi^{*} A_{T}$ and $L_{Z}=\pi^{*} L_{T}$. Thus, $M_{Z^{\prime}} \sim_{\mathbb{Q}} A_{Z^{\prime}}+L_{Z^{\prime}}$ where $A_{Z^{\prime}}$ is semi-ample and $L_{Z^{\prime}} \geq 0$. Perhaps after replacing $Z^{\prime}$ we may assume that $\operatorname{Supp}\left(B_{Z^{\prime}}+L_{Z^{\prime}}\right)$ has simple normal crossing singularities. By our choice of $\delta^{\prime}$, each coefficient of $B_{Z^{\prime}}$ is $\leq 1-\delta^{\prime}$.

Since $A_{T}+L_{T}$ is nef and $A_{T}$ is ample,

$$
A_{T}+\frac{1}{a+1} L_{T}=\frac{a}{a+1} A_{T}+\frac{1}{a+1}\left(A_{T}+L_{T}\right)
$$

is ample for any $a>0$. So, perhaps after replacing $L_{Z^{\prime}}$ with $\frac{a}{a+1} L_{Z^{\prime}}$ and $A_{Z^{\prime}}$ with $A_{Z^{\prime}}+\frac{1}{a+1} L_{Z^{\prime}}$ for some sufficiently small $a>0$, we may assume that the coefficients of $L_{Z^{\prime}}$ are sufficiently small. Thus, we can assume that the coefficients of $B_{Z^{\prime}}+L_{Z^{\prime}}$ are all $\leq 1-\delta$. Perhaps after replacing $A_{Z^{\prime}}$ we can also assume that $\operatorname{Supp}\left(B_{Z^{\prime}}+A_{Z^{\prime}}+L_{Z^{\prime}}\right)$ has simple normal crossing singularities and that the coefficients of $B_{Z^{\prime}}+A_{Z^{\prime}}+L_{Z^{\prime}}$ are $\leq 1-\delta$ and that $A_{Z^{\prime}} \geq 0$.

Let $A_{Z}, L_{Z}$ be the pushdown of $A_{Z^{\prime}}, L_{Z^{\prime}}$ respectively. Since

$$
K_{Z^{\prime}}+B_{Z^{\prime}}+A_{Z^{\prime}}+L_{Z^{\prime}}=g^{*}\left(K_{Z}+B_{Z}+A_{Z}+L_{Z}\right)
$$

we deduce that $\left(Z, B_{Z}+A_{Z}+L_{Z}\right)$ is a $\delta$-lc pair. By putting $M_{Z}:=A_{Z}+L_{Z}$ we finish the proof of the theorem when $B$ has rational coefficients.

Now we come to the general case, that is, when the coefficients of $B$ are not necessarily rational. We will do an approximation to reduce to the rational case. Pick $\epsilon^{\prime \prime} \in\left(0, \epsilon^{\prime}\right)$, let $\delta^{\prime \prime}>0$ be the number given by Proposition 3.5 for the data $d, \epsilon^{\prime \prime}, \mathcal{P}$, and pick some $\delta \in\left(0, \delta^{\prime \prime}\right)$. Then, using Shokurov's polytopes [19], we can find real numbers $r_{i} \geq 0$ and rational boundaries $B^{i}$ such that

- $\sum r_{i}=1$ and $K_{X}+B=\sum r_{i}\left(K_{X}+B^{i}\right)$,

- $K_{X}+B^{i} \sim_{\mathbb{Q}} 0 / Z$,

- $\operatorname{Supp} B^{i}=\operatorname{Supp} B$,

- $\left(X, B^{i}\right)$ are $\epsilon^{\prime \prime}$-lc.

Applying the arguments above in the rational case, for each $i$ we can choose $M_{Z}^{i} \geq 0$ such that

$$
K_{X}+B^{i} \sim_{\mathbb{Q}} f^{*}\left(K_{Z}+B_{Z}^{i}+M_{Z}^{i}\right)
$$

and that $\left(Z, B_{Z}^{i}+M_{Z}^{i}\right)$ is a $\delta$-lc pair.

Fix a prime divisor $D$ on $Z$ and let its coefficient in $B_{Z}$ and $B_{Z}^{i}$ be $b$ and $b^{i}$ respectively. By definition, the pair $\left(X, B^{i}+\left(1-b^{i}\right) f^{*} D\right)$ is lc over the generic point of $D$. But then the pair $\left(X, B+\sum r_{i}\left(1-b^{i}\right) f^{*} D\right)$ is lc over the generic 
point of $D$. This means that

$$
1-\sum r_{i} b^{i}=\sum r_{i}\left(1-b^{i}\right) \leq 1-b
$$

which in turn implies that $\sum r_{i} b^{i} \geq b$. In other words, $\sum r_{i} B_{Z}^{i} \geq B_{Z}$.

Now, the pair

$$
\left(Z, \sum r_{i} B_{Z}^{i}+\sum r_{i} M_{Z}^{i}\right)
$$

is $\delta$-lc and by putting

$$
M_{Z}:=\sum r_{i} B_{Z}^{i}+\sum r_{i} M_{Z}^{i}-B_{Z}
$$

we conclude that $\left(Z, B_{Z}+M_{Z}\right)$ is $\delta$-lc and

$$
K_{X}+B \sim_{\mathbb{R}} f^{*}\left(K_{Z}+B_{Z}+M_{Z}\right)
$$

This completes the proof of the theorem.

We need the following theorem for the proof of Corollary 1.4.

Theorem 4.1. Let $\epsilon, \lambda>0$ be real numbers. Consider the set of pairs $\left(F, B_{F}\right)$ introduced just before Corollary 1.4 with the extra assumption $d \leq 3$. Then, $\mathcal{R}$, the set of the couples ( F, Supp $\left.B_{F}\right)$ is a bounded family.

Proof. First assume that $\operatorname{dim} F=1$ which means that $F \simeq \mathbb{P}^{1}$. Replacing $\lambda$ with a smaller number we may assume that it is rational. Since $\operatorname{deg} B_{F}=2$ and each coefficient of $B_{F}$ is $\geq \lambda$, the number of components of Supp $B_{F}$ is bounded only depending on $\lambda$. Then, the set of the couples $\left(F, \operatorname{Supp} B_{F}\right)$ belongs to a bounded family using Hilbert schemes of zero-dimensional subschemes of $F$.

Now assume that $\operatorname{dim} F=2$. Let $\Delta_{F}$ be the boundary obtained from $B_{F}$ by replacing each coefficient with $\lambda$. By definition, $C_{F}:=B_{F}-\Delta_{F} \geq 0$. Pick a small number $t>0$ so that $\left(F, B_{F}+t C_{F}\right)$ is still klt. Run the LMMP on $K_{F}+B_{F}+t C_{F} \sim_{\mathbb{R}} t C_{F}$. We get a log minimal model on which the pushdown of $C_{F}$ is nef. By replacing $F$ with that model we could assume that $C_{F}$ is nef. Note that the LMMP can contract only the components of $B_{F}$ so we can pullback boundedness to the original setting.

By Alexeev [1] the varieties $F$ belong to a bounded family. In particular, the Cartier index of $K_{F}+\Delta_{F}$ is bounded. So, we can pick a $\mathbb{Q}$-divisor $A_{F} \geq 0$ such that $K_{F}+\Delta_{F}+A_{F} \sim_{\mathbb{Q}} 0$ and such that the coefficients of $\Delta_{F}+A_{F}$ belong to a fixed finite set depending only on $\epsilon$ and $\lambda$. Now, apply [10, Corollary 1.7] to get the boundedness of the couples $\left(F, \operatorname{Supp} \Delta_{F}\right)=\left(F, \operatorname{Supp} B_{F}\right)$.

Proof. (of Corollary 1.4) This follows from Theorems 4.1 and 1.3.

Remark 4.2 Actually, if one tries to prove the boundedness of $\mathcal{R}$ for $d>3$ inductively, then variants of Theorem 1.3 appear naturally in the induction process. More precisely: pick $\left(F, B_{F}\right)$ as defined just before Corollary 1.4. After running a suitable LMMP one can assume that $F$ has a Mori fibre space structure $F \rightarrow G$. Assume that $\operatorname{dim} G>0$. The couples associated to the $\log$ general fibres of this morphism are bounded by induction. One uses a variant of Theorem 1.3 to show that $G$ is also bounded (one needs a stronger form of 1.3 in which the coefficients of $B_{G}+M_{G}$ are not too small and this needs an effective 
version of Ambro's result on $M_{G}$ ) ; the next step is to use these boundedness results to prove that $F$ itself together with $\operatorname{Supp} B_{F}$ are bounded. This is more related to the work of Shokurov mentioned in the introduction. On the other hand, if $\operatorname{dim} G=0$, one needs different arguments.

Proof. (of Corollary 1.5) This is immediate by Theorem 1.3 and [10, Corollary 1.7].

Proof. (of Corollary 1.6) Let $(X, B)$ and $f: X \rightarrow Z$ be as in Conjecture $S_{d, \epsilon, \mathcal{P}}$ such that $d \leq \operatorname{dim} Z+1$. We may assume that $d=\operatorname{dim} Z+1$ otherwise $f$ is birational and we can argue as in the proof of Theorem 1.3. Using the arguments of the proof of Lemma 3.2, Proposition 3.5, and Theorem 1.3, we can reduce the problem to the case $\operatorname{dim} Z=1$ and $\operatorname{dim} X=2$. Moreover, we only need to show that the coefficients of $B_{Z}$ are $\leq 1-\delta$ for a fixed $\delta>0$ depending only on $\epsilon$.

By replacing $X$ with its minimal resolution, we may assume that $X$ is smooth. Next by running an LMMP/Z on $K_{X}$ we can assume that $X \rightarrow Z$ is an extremal contraction, that is, in this case a $\mathbb{P}^{1}$-bundle. Fix $\epsilon^{\prime} \in(0, \epsilon)$. Pick a closed point $D \in Z$ and let $t$ be the $\epsilon^{\prime}$-lc threshold of $f^{*} D$ with respect to the pair $(X, B)$, that is, $t$ is the largest number so that $\left(X, B+t f^{*} D\right)$ is $\epsilon^{\prime}$-lc. Here $T:=f^{*} D$ is a reduced curve. If the coefficient of $T$ in $B+t f^{*} D$ is $1-\epsilon^{\prime}$, then $t \geq\left(\epsilon-\epsilon^{\prime}\right)$ because the coefficient of $T$ in $B$ is at most $1-\epsilon$. In this case, the coefficient of $D$ in $B_{Z}$ is at most $1-\epsilon+\epsilon^{\prime}$ and $\delta=\epsilon-\epsilon^{\prime}$ works. From now on we assume that the coefficient of $T$ in $B+t f^{*} D$ is $<1-\epsilon^{\prime}$.

There is a prime exceptional $/ X$ divisor $E$ such that $a\left(E, X, B+t f^{*} D\right)=\epsilon^{\prime}$. Let $Y \rightarrow X$ be the extremal contraction which extracts $E$. There is another extremal ray on $Y / Z$ which we can contract to get $X^{\prime}$, and $X^{\prime} \rightarrow Z$ is an extremal contraction. However, $X^{\prime}$ may not be smooth. Let $g, h, f^{\prime}$ denote the contractions $Y \rightarrow X, Y \rightarrow X^{\prime}$, and $X^{\prime} \rightarrow Z$ respectively. Write $K_{X^{\prime}}+B^{\prime}=$ $h_{*} g^{*}\left(K_{X}+B\right)$ and let $T^{\prime}=\operatorname{Supp} f^{*} D$. Then, the coefficient of $T^{\prime}$ in $B^{\prime}+t f^{* *} D$ is $1-\epsilon^{\prime}$. Since the coefficient of $T^{\prime}$ in $B^{\prime}$ is at most $1-\epsilon$, it is enough to show that $f^{* *} D=m^{\prime} T^{\prime}$ where $m^{\prime}$ is bounded depending on $\epsilon$.

Applying the boundedness of $\epsilon^{\prime}$-lc complements in dimension two [5], we get a real number $\epsilon^{\prime \prime}>0$ and a finite set $\Lambda$ of rational numbers depending only on $\epsilon^{\prime}$ which satisfy: there is a boundary $\Delta^{\prime}$ such that $\left(X^{\prime}, \Delta^{\prime}\right)$ is $\epsilon^{\prime \prime}$-lc, $K_{X^{\prime}}+\Delta^{\prime} \sim_{\mathbb{Q}} 0 / Z$, and the coefficients of $\Delta^{\prime}$ belong to $\Lambda$. Now we can apply Corollary 1.4 to get the bound on $\mathrm{m}^{\prime}$.

Remark 4.3 An obvious question to ask is: what can we do about Conjecture $S_{d, \epsilon, \mathcal{P}}$ if $\mathcal{P}$ is not bounded? Let $(X, B)$ and $f: X \rightarrow Z$ be as in Conjecture $S_{d, \epsilon, \mathcal{P}}$, and let $\left(F, B_{F}\right)$ be a log general fibre. In some cases, the couples $\left(F, \operatorname{Supp} B_{F}\right)$ belong to a bounded family $\mathcal{P}^{\prime}$ even if $\mathcal{P}$ is not bounded. For example, all the couples in $\mathcal{P}$ of dimension $\leq d-1$ may belong to a bounded family $\mathcal{P}^{\prime}$, that is, the unbounded part of $\mathcal{P}$ may not be relevant to the conjecture. In this case, Conjecture $S_{d, \epsilon, \mathcal{P}^{\prime}}$ implies Conjecture $S_{d, \epsilon, \mathcal{P}}$, and we can use Theorem 1.3 to prove Conjecture $S_{d, \epsilon, \mathcal{P}^{\prime}}$. 
In general, we cannot shrink $\mathcal{P}$ to a bounded family $\mathcal{P}^{\prime}$. The idea then is to modify the pair $(X, B)$ to get boundedness. For example, as in the proof of Corollary 1.6, one can hope to find a real number $\epsilon^{\prime}>0$ and a fixed finite set $\Lambda$ of real numbers depending only on $d, \epsilon^{\prime}$ such that: there is a boundary $\Delta$ so that $(X, \Delta)$ is $\epsilon^{\prime}$-lc, $K_{X}+\Delta \sim_{\mathbb{Q}} 0 / Z$, and the coefficients of $\Delta$ belong to $\Lambda$. Next one applies Corollary 1.5. This is closely related to the theory of complements [20][5].

\section{REFERENCES}

[1] V. Alexeev; Boundedness and $K^{2}$ for log surfaces. Internat. J. Math. 5 (1994), no. 6, $779-810$.

[2] V. Alexeev, A. Borizov; On the Log Discrepancies in Mori Contractions. arXiv:1208.3271v1.

[3] F. Ambro; The moduli b-divisor of an lc-trivial fibration. Compos. Math. 141 (2005), no. $2,385-403$.

[4] F. Ambro; Basic properties of log canonical centers. arXiv:math/0611205v1.

[5] C. Birkar; Boundedness of epsilon-lc complements on surfaces. Available at https://www.dpmms.cam.ac.uk/ cb496/research.html.

[6] C. Birkar; Existence of log canonical flips and a special LMMP. Publ. Math. de l'IHÉS, Volume 115, Issue 1 (2012), 325-368.

[7] C. Birkar, P. Cascini, C. D. Hacon, J. McKernan; Existence of minimal models for varieties of log general type. J. Amer. Math. Soc. 23 (2010), 405-468.

[8] O. Fujino, Y. Gongyo; On canonical bundle formulae and subadjunctions. To appear in Michigan Math. J. arXiv:1009.3996v1.

[9] C. D. Hacon, J. McKernan, C. Xu; On the birational automorphisms of vari- eties of general type. arXiv:1011.1464v1, To appear, Ann. of Math.

[10] C. D. Hacon, J. MKernan, C. Xu; ACC for log canonical thresholds. arXiv:1208.4150v1.

[11] Y. Kawamata; Minimal models and the Kodaira dimension of algebraic fiber spaces. J. Reine Angew. Math. 363 (1985), 1-46.

[12] Y. Kawamata; Subadjunction of log canonical divisors for a variety of codimension 2, Contemporary Mathematics 207 (1997),79-88.

[13] Y. Kawamata; Subadjunction of log canonical divisors, II, Amer. J. Math. 120 (1998), 893-899.

[14] J. Kollár, S. Mori; Birational Geometry of Algebraic Varieties. Cambridge University Press (1998).

[15] L. Ein, R. Lazarsfeld, M. Mustaţă, M. Nakamaye, M. Popa: Asymptotic invariants of line bundles. Pure Appl. Math. Q. 1 (2005), 379-403.

[16] S. Mori and Yu. Prokhorov; On Q-conic bundles. Publ. Res. Inst. Math. Sci. 44 (2008), no. $2,315-369$.

[17] S. Mori and Yu. Prokhorov; Multiple fibers of del Pezzo fibrations. arXiv:0806.3809v1.

[18] V.V. Shokurov; Three-dimensional log fips. With an appendix in English by Yujiro Kawamata. Russian Acad. Sci. Izv. Math. 40 (1993), no. 1, 95-202.

[19] V.V. Shokurov; 3-fold log models. Algebraic geometry, 4. J. Math. Sci. 81 (1996), no. 3, 2667-2699.

[20] V.V. Shokurov; Complements on surfaces. Algebraic geometry, 10. J. Math. Sci. (New York) 102 (2000), no. 2, 3876-3932.

[21] V.V. Shokurov; Prelimiting flips. Proc. Steklov Inst. Math. 240 (2003), 75-213. 
DPMMS, Centre for Mathematical Sciences, Cambridge University, Wilberforce Road, Cambridge, CB3 0WB, UK

email: c.birkar@dpmms.cam.ac.uk 